\title{
Posttraumatic Stress Disorder and the Social Brain: Affect-Related Disruption of the Default and Mirror Networks
}

Kevin M. Tan, Lisa J. Burklund, Michelle G. Craske, Matthew D. Lieberman

University of California, Los Angeles

Department of Psychology

Corresponding author:

Matthew D. Lieberman

UCLA Psychology Department

1248 Franz Hall

Los Angeles, CA 90095-1563

Phone: 310.206 .4050

Email: lieber@ucla.edu

Short Title: PTSD and the Social Brain

Keywords: PTSD, mentalizing, theory of mind, action identification, default mode network, mirror neuron

Characters in Title: 112

Word Counts:

Abstract $=250$

Main text $=3980$

Number of Tables: 3

Number of Figures: 5

Supplementary Information: 1 table 


\section{PTSD AND THE SOCIAL BRAIN}

\section{Abstract}

Background: Social impairments, specifically in mentalizing and emotion recognition, are common and debilitating symptoms of posttraumatic stress disorder (PTSD). Despite this, little is known about the neural underpinnings of these impairments, as there have been no published neuroimaging investigations of social inference in PTSD.

Methods: Trauma-exposed veterans with and without PTSD ( $N=20$ each) performed the Why/How social inference task during functional magnetic resonance imaging (fMRI). The PTSD group had two fMRI sessions, between which they underwent affect labeling training. We probed the primary networks of the "social brain"-the default mode network (DMN) and mirror neuron system (MNS)-by examining neural activity evoked by mentalizing and action identification prompts, which were paired with emotional and non-emotional targets.

Results: Hyperactivation to emotional stimuli differentiated PTSD patients from controls, correlated with symptom severity, and predicted training outcomes. Critically, these effects were generally non-significant for non-emotional stimuli. PTSD-related effects were widely distributed throughout DMN and MNS. Effects were strongest in regions associated with the dorsal attention, ventral attention, and frontoparietal control networks. Unexpectedly, effects were non-significant in core affect regions.

Conclusions: The array of social cognitive processes subserved by DMN and MNS may be inordinately selective for emotional stimuli in PTSD. This selectivity may be tightly linked with attentional processes, as effects were strongest in attention-related regions. Putatively, we propose an attentional account of social inference dysfunction in PTSD, in which affective attentional biases drive widespread affect-selectivity throughout the social brain. This account aligns with numerous findings of affect-biased attentional processing in PTSD. 


\section{PTSD AND THE SOCIAL BRAIN}

\section{Introduction}

PTSD is characterized by intrusive trauma-related cognition (e.g. thoughts, dreams, and flashbacks), exaggerated affective responses (e.g. chronic fear, anxiety, and hyperarousal), and-conversely-affective blunting (e.g. anhedonia and emotional numbing) (1). Though PTSD is most commonly associated with affective dysfunction, social dysfunction is ubiquitous and often debilitating in PTSD (2-4). This has led many researchers to emphasize social and interpersonal factors in the development of PTSD and related adversity (5-7). A strong body of behavioral evidence links PTSD with deficits in emotion recognition (8-13) and mentalizing (13-17), a pattern of social cognitive impairment that is distinct from other anxiety disorders (18). Here, emotion recognition refers to perceiving and identifying others' emotions, while mentalizing refers to reasoning about others' mental states (e.g. beliefs, desires, and intentions). Emotion recognition can be considered a type of mentalizing, and both are facets of social inference and theory of mind (19-21). Taken together, there is converging behavioral evidence that social inference impairments are common and debilitating symptoms of PTSD. However, little is known about the neural underpinnings of these impairments.

In healthy populations, neuroimaging investigations have revealed that social inference is primarily subserved by two dissociable large-scale neural networks: the mirror neuron system (MNS) and default mode network (DMN) (22-24). MNS is associated with action identification, while DMN is associated with mentalizing (25-28). Mirror neurons, first discovered in macaque frontoparietal cortex, fire when actions are either performed or observed (29-31). In humans, similar sensorimotor "mirroring" responses may have been found in posterior inferior frontal gyrus (pIFG), dorsal premotor cortex (dPMC), inferior parietal sulcus (IPS), and lateral occipitotemporal cortex (LOTC) $(22,32)$. Such MNS regions have been shown to encode facial expressions (33), body language (34), and other goal-directed motor actions (35-37). During social inference, MNS is thought to represent observable sensorimotor actions (e.g. reaching 
for a cup) that are used by DMN to infer unobservable mental states and traits (e.g. thirsty) (27, 38, 39). Concordantly, MNS-based sensorimotor encoding appears to precede DMN-based mentalizing (40-43).

DMN regions are consistently recruited by mentalizing (44) and other functions that involve abstract mental state reasoning, such as theory of mind (45), emotion recognition (40), empathy (46), moral cognition (47), social working memory (48), and introspection (49). Though the anatomical distribution of these functions can differ, they often include the core DMN hubs of medial prefrontal cortex (mPFC), posterior cingulate cortex (PCC), and temporoparietal junction (TPJ) $(24,50-53)$. Aside from social and emotional functions, DMN is broadly associated with internally-oriented cognition (54). However, much of DMN activity occurs during rest, as DMN activation and connectivity are quickly engaged and sustained during the absence of goal-directed cognition (55-58). As such, DMN is widely thought to subserve the "default mode" of mammalian brain function $(23,58,59)$.

As detailed above, the neural substrates of social inference are fairly well-characterized in healthy populations. In contrast, little is known about the neurobiology of social inference impairments in PTSD, as there are currently no published neuroimaging studies that directly investigate social inference in PTSD. However, PTSD-related alterations in DMN activity have been found in other social tasks (60), such as script-driven social-emotional imagery (61-63), self-reference $(64,65)$, self-other reference $(66)$, and face perception $(67,68)$. Moreover, PTSDrelated differences in DMN connectivity are consistently found in resting-state studies (69-73). We are unaware of any reports of PTSD-related effects in regions explicitly defined as MNS. However, MNS appears to overlap substantially with the dorsal and ventral attention networks $(74,75,32,58)$, which are strongly implicated in PTSD-related attentional biases (76-86). Here, we perform the first neuroimaging investigation of social inference in PTSD. We probed activity in DMN and MNS regions due to their importance in social cognition and PTSD. 


\section{PTSD AND THE SOCIAL BRAIN}

To this end, we used functional magnetic resonance imaging (fMRI) to record brain activity during the Why/How social inference task (87) in trauma-exposed veterans with and without PTSD. The Why/How task contains mentalizing (Why) and action identification (How) prompts

(Figure 1), which robustly dissociate DMN and MNS activity (Figure 2) $(26,28,29,41,42,89)$. We explored whether DMN and MNS responses differentiate PTSD patients from controls, correlate with symptom severity, and predict training outcomes.

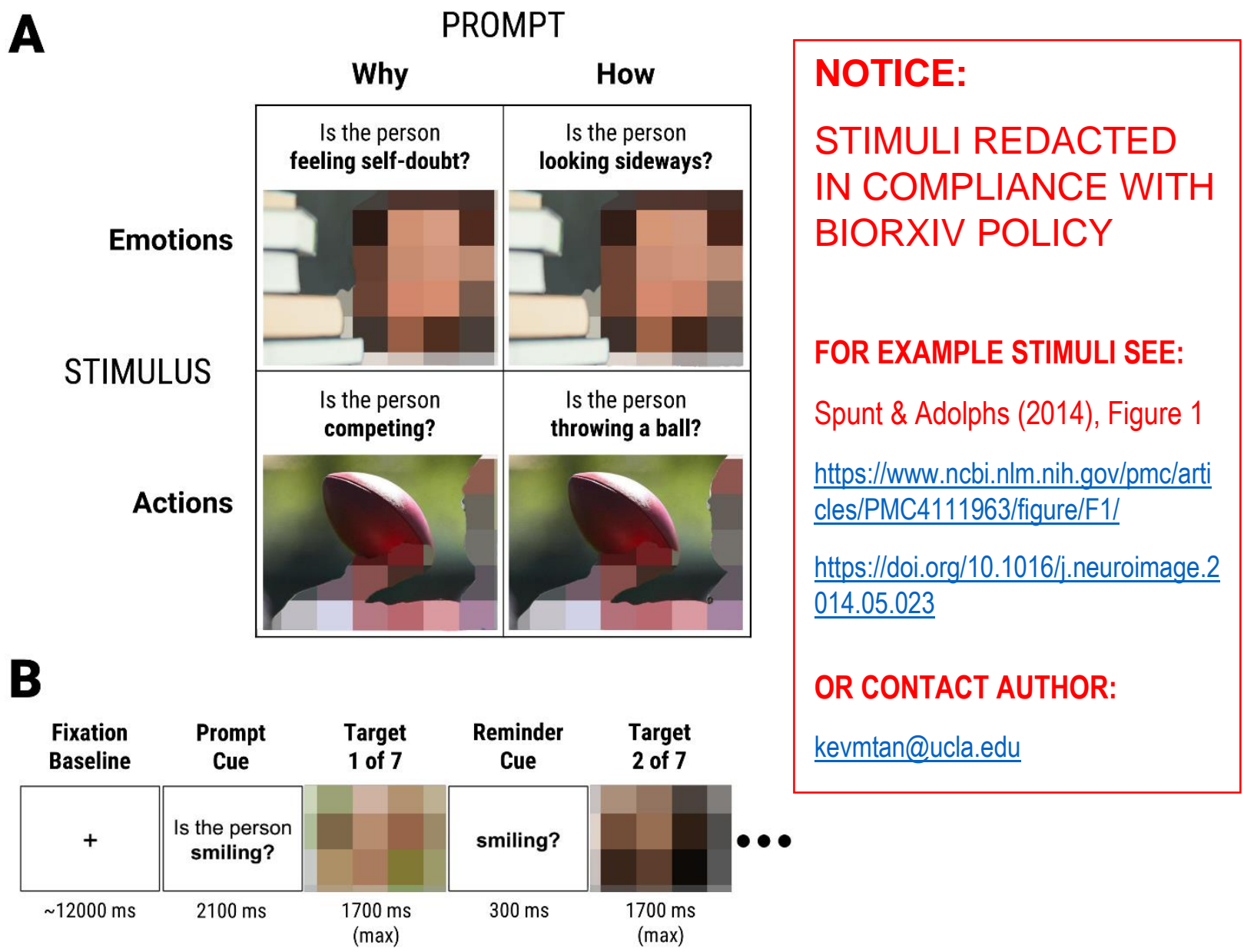

Figure 1. Summary of the standardized Why/How social inference task (Study 3 of Spunt \& Adolphs, 2014). (A) Diagram of the task's $2 \times 2$ design across Prompt and Stimulus. Each stimulus was shown twice: once with a mentalizing prompt (Why), and once with an action identification prompt (How). There were two types of stimuli: emotional facial expressions (Emotions), and intentional hand actions (Actions). (B) Sequence of events in a task block. Each block began with a prompt followed by seven target stimuli paired with that prompt. Participants were instructed to make true/false responses as quickly and accurately as possible during the presentation of target stimuli. Target stimuli were presented for $1700 \mathrm{~ms}$ or until a response was made. A reminder prompt was shown for $300 \mathrm{~ms}$ between target stimuli, and each block was preceded by a fixation baseline period. 


\section{Materials and Methods}

\section{Participants and Procedure}

Forty trauma-exposed military veterans were recruited from the Los Angeles area. All participants were exposed to combat trauma, mostly in Iraq and Afghanistan. Twenty participants met DSM-5 criteria for PTSD or other trauma-related disorder, while the control group $(N=20)$ had no current or lifetime psychiatric diagnoses. Diagnostic status was determined by the Clinician-Administered PTSD Scale (CAPS; 90), which was administered by certified research staff. Participants were 18-45 years old, English-speaking, right-handed, and were excluded for serious medical conditions, moderate-to-severe substance abuse, recent changes to medication/psychotherapy, chronic childhood abuse/neglect, and standard fMRI contraindications (e.g. metallic implants, claustrophobia, pregnancy). Participants provided informed consent, and the study was approved by the University of California, Los Angeles (UCLA) institutional review board.

All participants performed baseline pre-treatment assessments involving a clinical interview, questionnaires, and an fMRI scan. Only the PTSD group continued with three weeks of twice-weekly affect labeling training, followed by post-training assessments similar to the pretraining assessments. Affect labeling training involved repeated practice with several computerbased tasks that were designed to strengthen inhibitory capacity (90-93). This training regimen was investigated as proof-of-concept for a novel, brief computerized intervention for PTSD; full methods and data for affect labeling training will be presented in a separate manuscript (94).

In the baseline session, data from 18 PTSD and 17 control participants were used, while the rest were unusable due to lack of task data $(N=3)$, a previous brain tumor $(N=1)$, and noncompliance with fMRI instructions $(N=1)$. Due to participant dropout, only 13 PTSD participants completed the post-training session, and only data from 11 PTSD participants were used, while the rest were unusable due to lack of task data $(N=1)$, a previous brain tumor $(N=1)$, and 


\section{PTSD AND THE SOCIAL BRAIN}

suspected cannabis intoxication $(N=1)$.

\section{Why/How Social Inference Task}

Participants completed the "fast" version of the standardized Why/How social inference task (95), which corresponds to Study 3 in (87). The task features a $2 \times 2$ design across $\operatorname{Prompt}_{[\text {Why, How] }}$ and Stimulus [Emotions, Actions] $_{\text {(Figure 1A). Stimuli were photographs of emotional }}$ facial expressions (Emotions) or intentional hand actions (Actions). Each stimulus was shown twice, once with each prompt type. Why prompts involve mentalizing (e.g. "Is the person competing?), while How prompts involve action identification (e.g. "Is the person throwing a ball?"). Thus, there were four conditions: WhyEmotions, WhyActions, HowEmotions, and HowActions. The task was organized into 16 blocks, four blocks per condition. Blocks began with a text prompt followed by seven target stimuli paired with that prompt (Figure 1B). Participants were instructed to judge whether a prompt was true or false for a target stimulus as quickly and accurately as possible. The task was implemented in PsychophysicsToolbox 3 (96) running on MATLAB 2007a (97). The task was shown in the fMRI scanner via virtual reality goggles at $800 \times 600$ resolution, and responses were made through a button box held with the right hand.

\section{fMRI Acquisition and Preprocessing}

fMRI data were acquired at the UCLA Staglin Center for Cognitive Neuroscience using a Siemens TimTrio 3-Tesla MRI scanner. Functional data were collected through T2*-weighted echo-planar image volumes (slice thickness $=3 \mathrm{~mm}$, gap $=1 \mathrm{~mm}, 36$ slices, $\mathrm{TR}=2000 \mathrm{~ms}, \mathrm{TE}=$ $25 \mathrm{~ms}$, flip angle $=90^{\circ}$, matrix $=64 \times 64$, FOV $=200 \mathrm{~mm}$ ). Two structural scans were acquired: a matched-bandwidth T2-weighted anatomical scan (MBW; slice thickness = $4 \mathrm{~mm}$, no gap, 34 slices, $\mathrm{TR}=5000 \mathrm{~ms}, \mathrm{TE}=34 \mathrm{~ms}$, flip angle $=90^{\circ}$, matrix $=128 \times 128, \mathrm{FOV}=196 \mathrm{~mm}$ ), and a T1weighted, magnetization-prepared, rapid-acquisition, gradient-echo scan (MPRAGE; slice 


\section{PTSD AND THE SOCIAL BRAIN}

thickness $=1 \mathrm{~mm}$, gap $=.5 \mathrm{~mm}, 160$ slices, $\mathrm{TR}=1900 \mathrm{~ms}, \mathrm{TE}=3.43 \mathrm{~ms}$, flip angle $=9^{\circ}$, matrix $=$ $256 \times 256, \mathrm{FOV}=256 \mathrm{~mm})$.

fMRI data were preprocessed via SPM12 (98) and the DARTEL pipeline (99). For each subject and session, functional images were realigned and resliced to the mean functional image to correct for head motion. Then, the MBW was coregistered to the mean functional image, then the MPRAGE was coregistered to the MBW. Afterwards, the MPRAGE was segmented and bias-corrected. The resulting MPRAGE images and segmentation parameters were used to create a sample-specific image template, which was subsequently affineregistered into Montreal Neurological Institute (MNI) space (100). Deformation fields generated in the previous step were used to normalize all images into MNI space, with functional images undergoing integrated spatial smoothing ( $8 \mathrm{~mm}$, Gaussian kernel, full-width at half-maximum).

\section{Single-Subject fMRI Analysis}

To estimate neural responses to the Why/How task within each participant and session, task timings were specified in SPM12's general linear model and convolved with the canonical double-gamma hemodynamic response function. Realignment parameters were used as nuisance regressors to dampen the impact of remaining motion artifacts. Data were high-pass filtered at $1 / 128 \mathrm{~Hz}$ to correct for signal drift. Parameter estimates from Why-How contrasts for both stimulus types (WhyEmotions-HowEmotions and WhyActions-HowActions) were used for all subsequent group-level fMRI analyses.

\section{Group-Level Analyses}

Unless otherwise noted, group-level statistical analyses were performed via Matlab 2016b Statistics and Machine Learning Toolbox (101), with linear mixed-effects models (LMEMs) used for hypothesis testing. LMEMs were specified with the maximal random (withinsubject) effects structure justified by each analysis, as this has been shown to be ideal for 


\section{PTSD AND THE SOCIAL BRAIN}

hypothesis testing $(102,103)$. Post-hoc simple effects tests were performed for LMEMs with significant interaction effects. To obtain canonical "main effects," effects coding was used in multi-factor models with at least one categorical factor, otherwise dummy coding was used (104).

\section{Behavioral Analyses}

In the Why/How task, pre-training group differences in response time and accuracy were analyzed via standard and logistic LMEMs, respectively. Both LMEMs featured a full-factorial

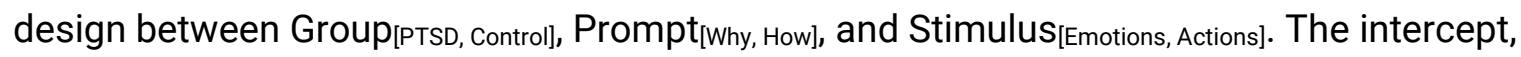
Prompt, Stimulus, and Prompt x Stimulus were nested within Subject.

\section{Region of Interest (ROI) fMRI Analyses}

To interrogate brain regions that subserve social inference, masks of a priori ROIs (Figure 2) were functionally defined by the Why-How contrast in an independent dataset featuring healthy participants $(N=50$; studies 1 and 3 in (87)). A one-sample two-tailed $t$-test was used to reveal brain regions that are differentially modulated by the Why and How conditions. The whole-network DMN (Why > How) and MNS (How > Why) masks (Figure 2A) were defined with a threshold of $p<0.001$. A more stringent threshold of $p<1 \times 10^{-6}$ was used to define ROIs within DMN and MNS (Figure 2B) that are thought to be key nodes of each network $(75,105)$

The masks obtained above were used to extract ROI parameter estimates (mean value of all voxels in a mask) from the single-subject/session Why-How contrasts in the present study. Multiple comparisons across ROls were accounted for by controlling the false discovery rate $(\mathrm{FDR})<0.05(106)$, and $p$-values were adjusted accordingly $\left(p_{\mathrm{FDR}}\right)$ through the procedure in (107). Pre-training group differences in neural response were analyzed in a LMEM with Group, Stimulus, and their interaction as effects; the intercept and Stimulus were nested within Subject. 
bioRxiv preprint doi: https://doi.org/10.1101/527176; this version posted January 23, 2019. The copyright holder for this preprint (which was not certified by peer review) is the author/funder. All rights reserved. No reuse allowed without permission.

PTSD AND THE SOCIAL BRAIN

In PTSD patients, the relationship between symptom severity (CAPS) and neural response was examined in a LMEM with CAPS and CAPS $\times$ Session $\times$ Stimulus effects; the intercept, Session, Stimulus, and Session x Stimulus were nested within Subject. The relationship between training outcome (Post-Pre CAPS score difference; CAPSdiff) and neural response was analyzed in a LMEM with CAPSdiff and CAPSdiff $x$ Stimulus as effects; the intercept and Stimulus were nested within Subject.
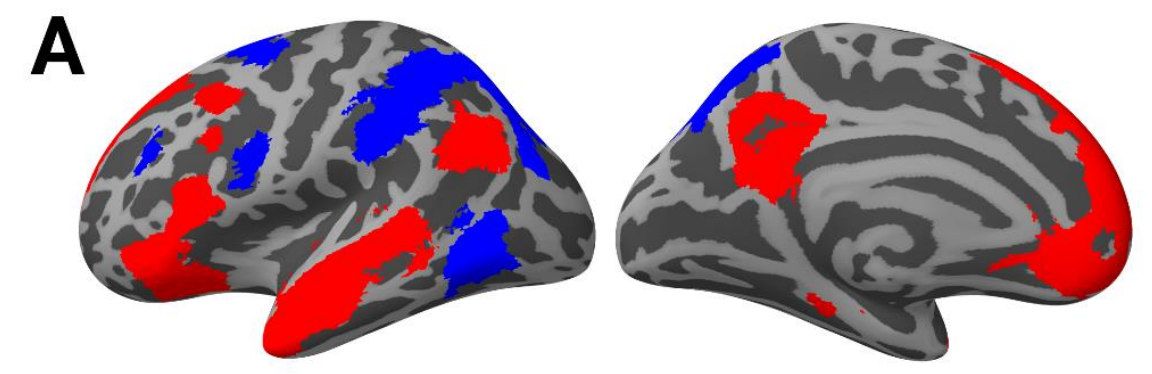

ROI (contrast)

DMN (Why > How)

MNS (How > Why)

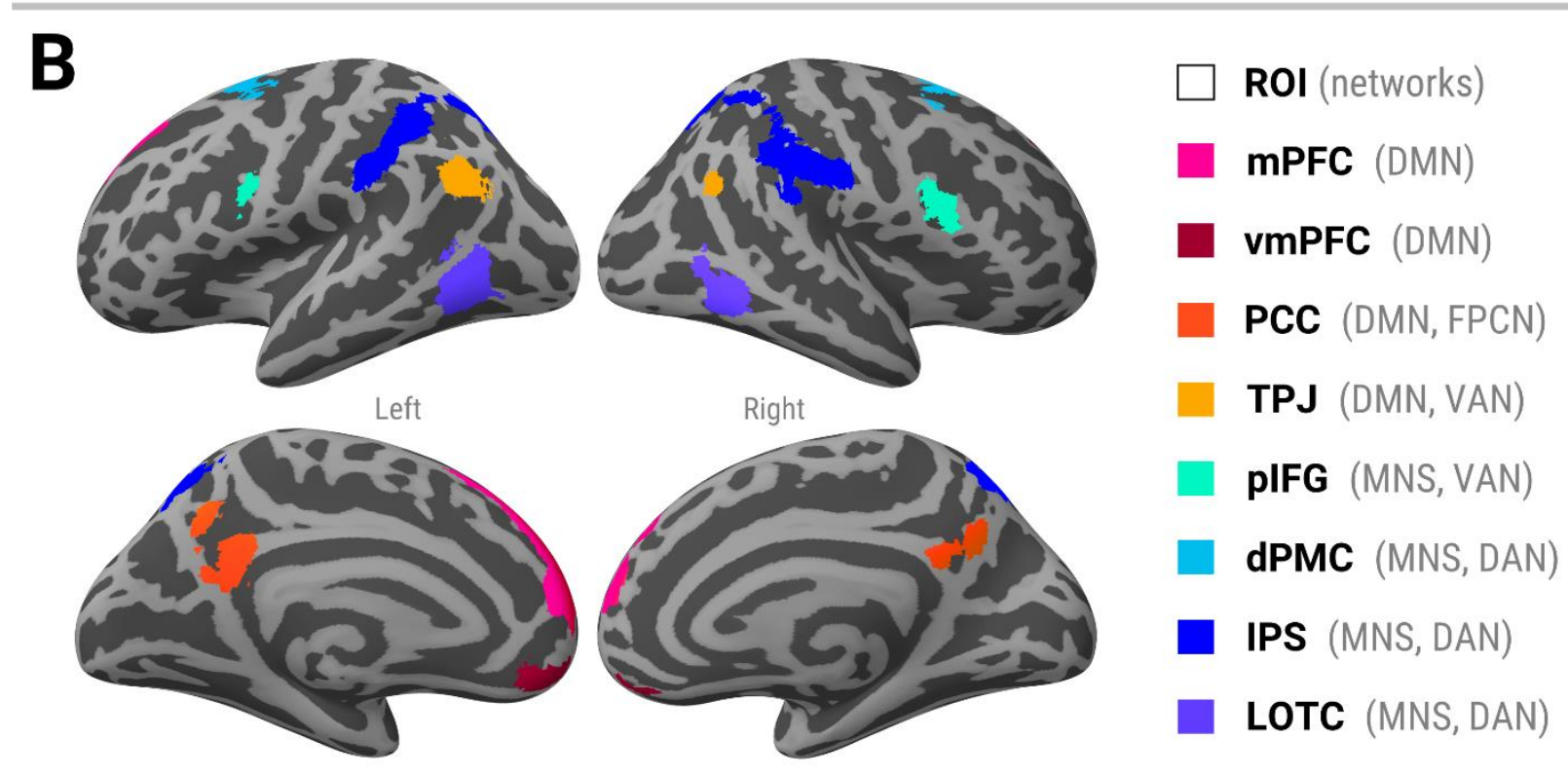

Figure 2. A priori ROI masks defined by the Why-How contrast in an independent dataset (Spunt \& Adolphs, 2014). The Why-How contrast dissociates DMN and MNS regions that are selective for mentalizing and action identification, respectively. All ROI masks are bilateral. (A) Whole-network DMN and MNS masks. (B) Within-network ROls that are thought to be key nodes of either the DMN and MNS. Some of these ROIs are also considered to be nodes of the attentional networks. Abbreviations: $\mathrm{ROI}=$ region of interest; $\mathrm{DMN}=$ default mode network; $\mathrm{MNS}=$ mirror-neuron system; $\mathrm{mPFC}=$ medial prefrontal cortex; $\mathrm{vmPFC}=$ ventromedial prefrontal cortex; $\mathrm{PCC}=$ posterior cingulate cortex; $\mathrm{TPJ}=$ temporoparietal junction; $\mathrm{pIFG}=$ posterior inferior frontal gyrus; $\mathrm{dPMC}=$ dorsal premotor cortex; IPS = intraparietal sulcus; LOTC = lateral occipitotemporal cortex; FPCN = frontoparietal control network; VAN = ventral attention network; DAN = dorsal attention network 


\section{PTSD AND THE SOCIAL BRAIN}

\section{Whole-brain fMRI Analyses}

Whole-brain group fMRI analyses were performed to complement the primary ROI analyses. Whole-brain group differences (pre-training) were examined using Aaron Schultz's MR Tools (108). We specified a general linear model with Group, Stimulus, and their interaction as effects, with the intercept and Stimulus nested within Subject. Residuals from this model were used in AFNI's 3dFWHMx and 3dClustSim (109) to estimate a cluster extent $(k)$ that controls familywise error rate $($ FWER $)<0.05$. Post-hoc simple effects tests were conducted in clusters with a significant interaction effect $(p<0.005, k>120$ voxels).

\section{Results}

Behavioral Results

The PTSD group featured greater symptom severity (CAPS score) than controls, and symptom severity was marginally reduced after affect labeling training. Full clinical results will be presented in a separate manuscript (94).

Unexpectedly, Why/How task performance (pre-training) did not differ significantly across PTSD and controls. For both response time and accuracy, the main effect of Group and all Group-related interaction effects were not significant (Supplemental Table S1).

Pre-training Neural Responses across the PTSD and Control Groups

Overall, the Why-How contrast produced activations in DMN ROIs (Figure 3A) and deactivations in MNS ROIs (Figure 3B), aligning with previous studies (87). Unexpectedly, the main effect of Group was not significant in any ROI. Instead, the Group x Stimulus interaction was significant in 4/5 DMN ROIs and 4/5 MNS ROIs. Within these ROIs, post-hoc tests revealed that only emotional expressions, not intentional actions, elicited significant Group differences. Specifically, Emotions evoked greater activation in the PTSD group relative to controls (Table 1). 
Table 1. Pre-training Why-How neural responses across Group and Stimulus

\begin{tabular}{|c|c|c|c|c|c|c|c|c|c|c|}
\hline \multirow[b]{2}{*}{ Effect } & \multicolumn{5}{|c|}{ DMN ROIs } & \multicolumn{5}{|c|}{ MNS ROIs } \\
\hline & ROI & $\boldsymbol{b}$ & SE & $t$ & $p_{\mathrm{FDR}}$ & ROI & $\boldsymbol{b}$ & SE & $t$ & $p_{\mathrm{FDR}}$ \\
\hline GroupPTSD-Control & \multirow[t]{5}{*}{ DMN } & 0.079 & 0.052 & 1.505 & 0.238 & \multirow[t]{5}{*}{ MNS } & 0.118 & 0.062 & 1.897 & 0.155 \\
\hline Stimulus Emotions-Actions & & -0.034 & 0.072 & -0.476 & 0.716 & & -0.035 & 0.074 & -0.464 & 0.716 \\
\hline Group x Stimulus & & 0.194 & 0.072 & 2.715 & 0.014 & & 0.198 & 0.074 & 2.656 & 0.014 \\
\hline Group (Emotions) $^{\dagger}$ & & 0.546 & 0.191 & 2.861 & 0.011 & & 0.633 & 0.207 & 3.054 & 0.009 \\
\hline Group (Actions) ${ }^{\dagger}$ & & -0.232 & 0.162 & -1.426 & 0.387 & & -0.159 & 0.181 & -0.878 & 0.387 \\
\hline GroupPTSD-Control & \multirow[t]{5}{*}{ mPFC } & 0.079 & 0.098 & 0.808 & 0.422 & \multirow[t]{5}{*}{ plFG } & 0.099 & 0.076 & 1.297 & 0.249 \\
\hline StimulusEmotions-Actions & & -0.010 & 0.069 & -0.138 & 0.891 & & -0.090 & 0.093 & -0.970 & 0.671 \\
\hline Group x Stimulus & & 0.213 & 0.069 & 3.073 & 0.010 & & 0.200 & 0.093 & 2.162 & 0.043 \\
\hline Group (Emotions) ${ }^{\dagger}$ & & 0.583 & 0.227 & 2.571 & 0.017 & & 0.599 & 0.251 & 2.383 & 0.023 \\
\hline Group (Actions) ${ }^{\dagger}$ & & -0.268 & 0.251 & -1.065 & 0.387 & & -0.203 & 0.228 & -0.888 & 0.387 \\
\hline GroupPTSD-Control & \multirow[t]{5}{*}{ vmPFC } & 0.088 & 0.063 & 1.400 & 0.238 & \multirow[t]{5}{*}{$\mathrm{dPMC}$} & 0.071 & 0.083 & 0.862 & 0.422 \\
\hline Stimulus Emotions-Actions & & -0.042 & 0.078 & -0.541 & 0.716 & & -0.126 & 0.074 & -1.716 & 0.602 \\
\hline Group x Stimulus & & 0.149 & 0.078 & 1.899 & 0.069 & & 0.207 & 0.074 & 2.817 & 0.014 \\
\hline Group (Emotions) $^{\dagger}$ & & \multicolumn{4}{|c|}{ Interaction NS } & & 0.557 & 0.210 & 2.654 & 0.016 \\
\hline Group (Actions) ${ }^{\dagger}$ & & \multicolumn{4}{|c|}{ Interaction NS } & & -0.272 & 0.233 & -1.168 & 0.387 \\
\hline Group $_{\text {PTSD-Control }}$ & \multirow[t]{5}{*}{ PCC } & 0.122 & 0.087 & 1.407 & 0.238 & \multirow[t]{5}{*}{ IPS } & 0.135 & 0.070 & 1.919 & 0.155 \\
\hline Stimulus $_{\text {Emotions-Actions }}$ & & -0.062 & 0.087 & -0.719 & 0.716 & & -0.107 & 0.091 & -1.183 & 0.602 \\
\hline Group x Stimulus & & 0.279 & 0.087 & 3.221 & 0.010 & & 0.249 & 0.091 & 2.750 & 0.014 \\
\hline Group (Emotions) $^{\dagger}$ & & 0.801 & 0.249 & 3.211 & 0.008 & & 0.769 & 0.233 & 3.296 & 0.008 \\
\hline Group (Actions) ${ }^{\dagger}$ & & -0.314 & 0.240 & -1.308 & 0.387 & & -0.228 & 0.226 & -1.010 & 0.387 \\
\hline GroupPTSD-Control & \multirow[t]{5}{*}{ TPJ } & 0.136 & 0.059 & 2.302 & 0.148 & \multirow[t]{5}{*}{ LOTC } & 0.146 & 0.066 & 2.223 & 0.148 \\
\hline StimulusEmotions-Actions & & -0.118 & 0.090 & -1.301 & 0.602 & & 0.130 & 0.083 & 1.554 & 0.602 \\
\hline Group x Stimulus & & 0.315 & 0.090 & 3.488 & 0.009 & & 0.134 & 0.083 & 1.613 & 0.111 \\
\hline Group (Emotions) $^{\dagger}$ & & 0.902 & 0.213 & 4.238 & 0.001 & & \multicolumn{4}{|c|}{ Interaction NS } \\
\hline Group (Actions) ${ }^{\dagger}$ & & -0.359 & 0.219 & -1.638 & 0.387 & & & Interac & on NS & \\
\hline
\end{tabular}

${ }^{\dagger}$ post-hoc simple effects test; $p_{\mathrm{FDR}}=p$-value adjusted for false discovery rate $<0.05 ; N S=$ not significant $\left(p_{\mathrm{FDR}}>0.05\right)$

Mirroring the ROI results, the whole-brain analysis (Figure $3 \mathrm{C}$ ) did not find the main effects of Group and Stimulus to be significant $(p<0.005$, clusterwise FWER $<0.05)$. Instead, Group x Stimulus was significant in 3 clusters (peak coordinates listed): left dorsolateral prefrontal cortex (dIPFC; $\left.x=-33, y=28, z=40, F_{1,33}=18.96, k=503\right)$, bilateral dorsal somatomotor cortices (dSMC; $x=-6, y=-28, z=60, F_{1,33}=16.73, k=811$ ), and left temporoparietal junction (TPJ; $x=-51, y=-51, z=39, F_{1,33}=16.40, k=476$ ). In all three clusters, post-hoc tests revealed that only emotional expressions, not intentional actions, elicited 


\section{PTSD AND THE SOCIAL BRAIN}

significant Group differences. Specifically, emotional expressions evoked greater activation in PTSD relative to controls in left dIPFC (Emotions: $t_{33}=3.60, p<0.001$; Actions: $t_{33}=-1.89, p=$ 0.07), dSMC (Emotions: $t_{33}=3.35, p=0.002$; Actions: $t_{33}=-1.94, p=0.06$ ), and left TPJ

(Emotions: $t_{33}=4.04, p<0.001$; Actions: $t_{33}=-1.98, p=0.06$ ).
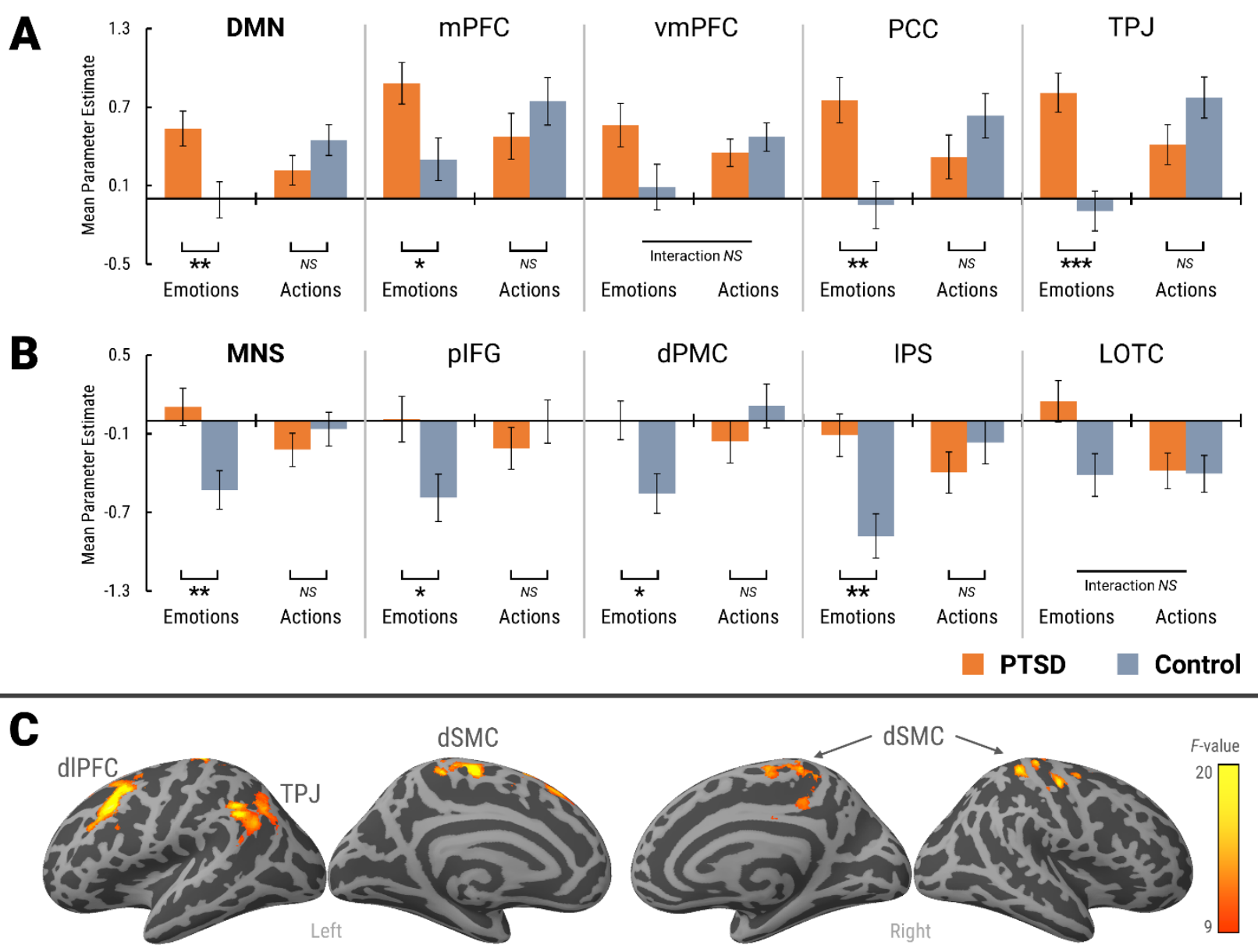

Figure 3. Pre-training Why-How neural responses featured a Group x Stimulus interaction. (A, B) Mean parameter estimates across Group and Stimulus in all (A) DMN and (B) MNS ROls. Error bars represent standard error of the mean. U-shaped brackets indicate the significance of Group simple effects. (C) Whole-brain analysis of the Group x Stimulus interaction $(p<0.005$, clusterwise FWER $<0.05)$.

Abbreviations: dIPFC $=$ dorsolateral prefrontal cortex; $\mathrm{dSMC}=$ dorsal somatomotor cortices; FWER $=$ familywise error rate; ${ }^{*} p_{\mathrm{FDR}}<0.05 ;{ }^{* \star} p_{\mathrm{FDR}}<0.01 ;{ }^{* \star \star} p_{\mathrm{FDR}}<0.001 ;{ }^{N S} p_{\mathrm{FDR}}>0.05$.

\section{Relationship between Symptom Severity and Neural Responses (PTSD only)}

Figure 4 and Table 2 show the relationship between symptom severity and Why-How neural responses in PTSD patients. The main effect of CAPS score was not significant in any 


\section{PTSD AND THE SOCIAL BRAIN}

ROI. Instead, the CAPS $\times$ Session $x$ Stimulus interaction was significant in 4/5 DMN ROIs and 4/5 MNS ROI. Post-hoc CAPS simple effects tests revealed that the pattern of interaction was consistent across these ROIs. For emotional stimuli, the CAPS correlation was positive during pre-training (significant in PCC and IPS), and negative during post-training (significant in pIFG).

For action stimuli, the CAPS correlation was negative during pre-training (not significant in any ROI), and positive during post-training (significant in DMN, MNS, and IPS).
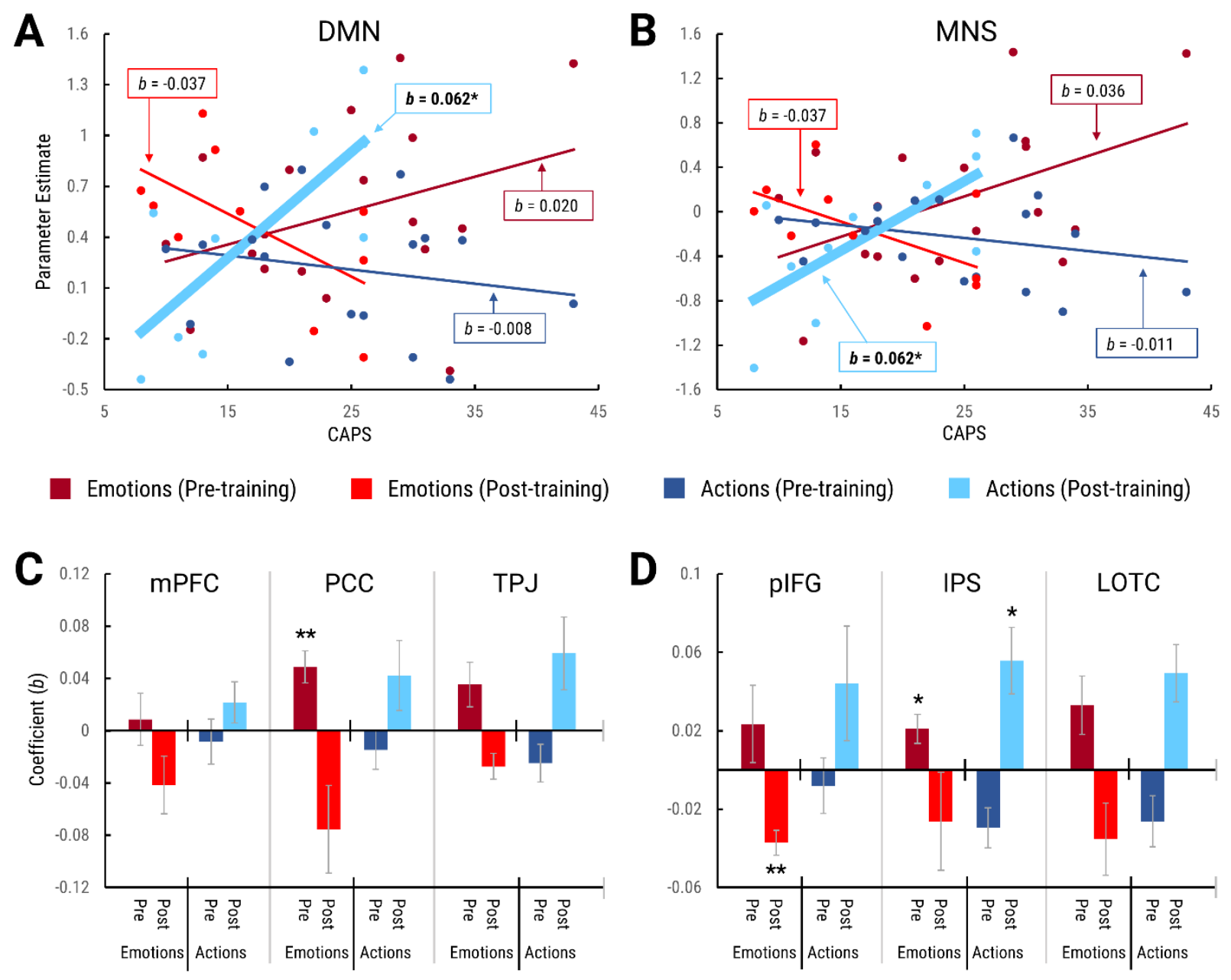

Figure 4. Relationship between symptom severity (CAPS) and Why-How neural responses in PTSD patients. Only ROIs with a significant CAPS $x$ Session $x$ Stimulus interaction are shown. The top panels show scatterplots of parameter estimates and CAPS scores in the (A) DMN and (B) MNS whole-network masks, with regression lines for CAPS simple effects. Thick lines represent significant regression coefficients, while thin lines represent non-significant regression coefficients. The bottom panels show CAPS simple effects regression coefficients for ROIs within (C) DMN and (D) MNS. Error bars represent standard error of regression coefficients. Abbreviations: CAPS = Clinician-Administered PTSD Scale 


\section{PTSD AND THE SOCIAL BRAIN}

Table 2. Relationship between symptom severity (CAPS) and Why-How neural activation in PTSD patients

\begin{tabular}{|c|c|c|c|c|c|c|c|c|c|c|}
\hline \multirow[b]{2}{*}{ Effect } & \multicolumn{5}{|c|}{ DMN ROIs } & \multicolumn{5}{|c|}{ MNS ROIs } \\
\hline & ROI & $\boldsymbol{b}$ & SE & $t$ & $p_{\mathrm{FDR}}$ & ROI & $b$ & SE & $t$ & $p_{\mathrm{FDR}}$ \\
\hline$\overline{\text { CAPS }}$ & $\overline{D M N}$ & 0.000 & 0.004 & -0.099 & 0.986 & MNS & 0.001 & 0.005 & 0.181 & 0.986 \\
\hline CAPS $x$ Session $x$ Stimulus & & -0.011 & 0.002 & -5.683 & $<0.001$ & & -0.007 & 0.003 & -2.823 & 0.010 \\
\hline CAPS (Pre, Emotions) $^{\dagger}$ & & 0.020 & 0.013 & 1.559 & 0.185 & & 0.036 & 0.016 & 2.250 & 0.083 \\
\hline CAPS (Post, Emotions) $^{\dagger}$ & & -0.008 & 0.010 & -0.837 & 0.554 & & -0.012 & 0.010 & -1.147 & 0.530 \\
\hline CAPS (Pre, Actions) ${ }^{\dagger}$ & & -0.037 & 0.015 & -2.451 & 0.106 & & -0.037 & 0.018 & -2.126 & 0.106 \\
\hline CAPS (Post, Actions) ${ }^{\dagger}$ & & 0.063 & 0.017 & 3.702 & 0.045 & & 0.063 & 0.021 & 2.986 & 0.046 \\
\hline CAPS & ك & -0.001 & 0.009 & -0.161 & 0.986 & pIFG & -0.017 & 0.006 & -2.715 & 0.089 \\
\hline CAPS $x$ Session $x$ Stimulus & & -0.011 & 0.003 & -3.529 & 0.002 & & -0.005 & 0.002 & -2.108 & 0.050 \\
\hline CAPS (Pre, Emotions) $^{\dagger}$ & & 0.009 & 0.020 & 0.435 & 0.670 & & 0.023 & 0.020 & 1.185 & 0.289 \\
\hline CAPS (Post, Emotions) ${ }^{\dagger}$ & & -0.008 & 0.017 & -0.477 & 0.640 & & -0.008 & 0.014 & -0.569 & 0.640 \\
\hline CAPS (Pre, Actions) $^{\dagger}$ & & -0.042 & 0.022 & -1.877 & 0.111 & & -0.037 & 0.006 & -5.882 & 0.003 \\
\hline CAPS (Post, Action & & 0.022 & 0.016 & 1.375 & 0.206 & & 0.044 & 0.029 & 1.511 & 0.193 \\
\hline CAPS & vmPFC & 0.003 & 0.006 & 0.548 & 0.986 & $\mathrm{dPMC}$ & -0.007 & 0.007 & -1.074 & 0.719 \\
\hline CAPS $x$ Session $x$ Stimulus & & -0.004 & 0.003 & -1.495 & 0.157 & & -0.004 & 0.003 & -1.431 & 0.158 \\
\hline CAPS (Pre, Emotions) ${ }^{\dagger}$ & & & Interac & ction NS & & & & Interac & ction NS & \\
\hline CAPS (Post, Emotions) ${ }^{\dagger}$ & & & Interac & ction NS & & & & Interac & ction NS & \\
\hline CAPS (Pre, Actions) ${ }^{\dagger}$ & & & Interac & ction NS & & & & Interac & ction NS & \\
\hline CAPS (Post, Actions) ${ }^{\dagger}$ & & & Interac & ction NS & & & & Intera & ction NS & \\
\hline CAPS & PCC & 0.013 & 0.006 & 2.225 & 0.152 & IPS & 0.010 & 0.005 & 1.796 & 0.261 \\
\hline CAPS $x$ Session $x$ Stimulus & & -0.011 & 0.003 & -3.093 & 0.006 & & -0.008 & 0.003 & -3.041 & 0.006 \\
\hline CAPS (Pre, Emotions) ${ }^{\dagger}$ & & 0.049 & 0.012 & 3.977 & 0.009 & & 0.021 & 0.007 & 2.847 & 0.047 \\
\hline CAPS (Post, Emotions) ${ }^{\dagger}$ & & -0.015 & 0.015 & -1.002 & 0.530 & & -0.030 & 0.010 & -2.899 & 0.084 \\
\hline CAPS (Pre, Actions) ${ }^{\dagger}$ & & -0.075 & 0.034 & -2.248 & 0.106 & & -0.026 & 0.025 & -1.051 & 0.324 \\
\hline CAPS (Post, Actions) ${ }^{\dagger}$ & & 0.042 & 0.027 & 1.573 & 0.193 & & 0.056 & 0.017 & 3.277 & 0.045 \\
\hline CAPS & TPJ & 0.000 & 0.005 & -0.018 & 0.986 & LOTC & -0.003 & 0.005 & -0.488 & 0.986 \\
\hline CAPS x Session x Stimulus & & -0.010 & 0.002 & -4.075 & 0.001 & & -0.009 & 0.002 & -4.520 & $<0.001$ \\
\hline CAPS (Pre, Emotions) $^{\dagger}$ & & 0.035 & 0.017 & 2.091 & 0.085 & & 0.033 & 0.015 & 2.219 & 0.083 \\
\hline CAPS (Post, Emotions) ${ }^{\dagger}$ & & -0.025 & 0.014 & -1.714 & 0.282 & & -0.026 & 0.013 & -2.014 & 0.244 \\
\hline CAPS (Pre, Actions) ${ }^{\dagger}$ & & -0.027 & 0.010 & -2.761 & 0.098 & & -0.035 & 0.018 & -1.913 & 0.111 \\
\hline CAPS (Post, Actions) ${ }^{\dagger}$ & & 0.059 & 0.028 & 2.131 & 0.105 & & 0.049 & 0.020 & 2.492 & 0.075 \\
\hline
\end{tabular}

${ }^{\dagger}$ post-hoc simple effects test 


\section{PTSD AND THE SOCIAL BRAIN}

Predicting Training Outcomes from Pre-Training Neural Responses (PTSD only)

Figure 5 and Table 3 show the relationship between training outcomes and pre-training Why-How neural responses for PTSD participants who completed affect labeling training. The main effect of Post-Pre CAPS score difference (CAPSdiff) was not significant in any ROI. Instead, CAPSdiff x Stimulus was significant in 3/5 DMN ROls and 5/5 MNS ROls. Post-hoc CAPSdiff simple effects tests revealed that the pattern of interaction was consistent across these ROIs. The CAPSdiff correlation was negative for emotional stimuli (significant in DMN, PCC, TPJ, MNS, IPS, and LOTC) and flat for action stimuli (not significant in any ROI). In sum, larger neural responses to emotional stimuli predicted larger decreases in symptom severity after affect labeling training.
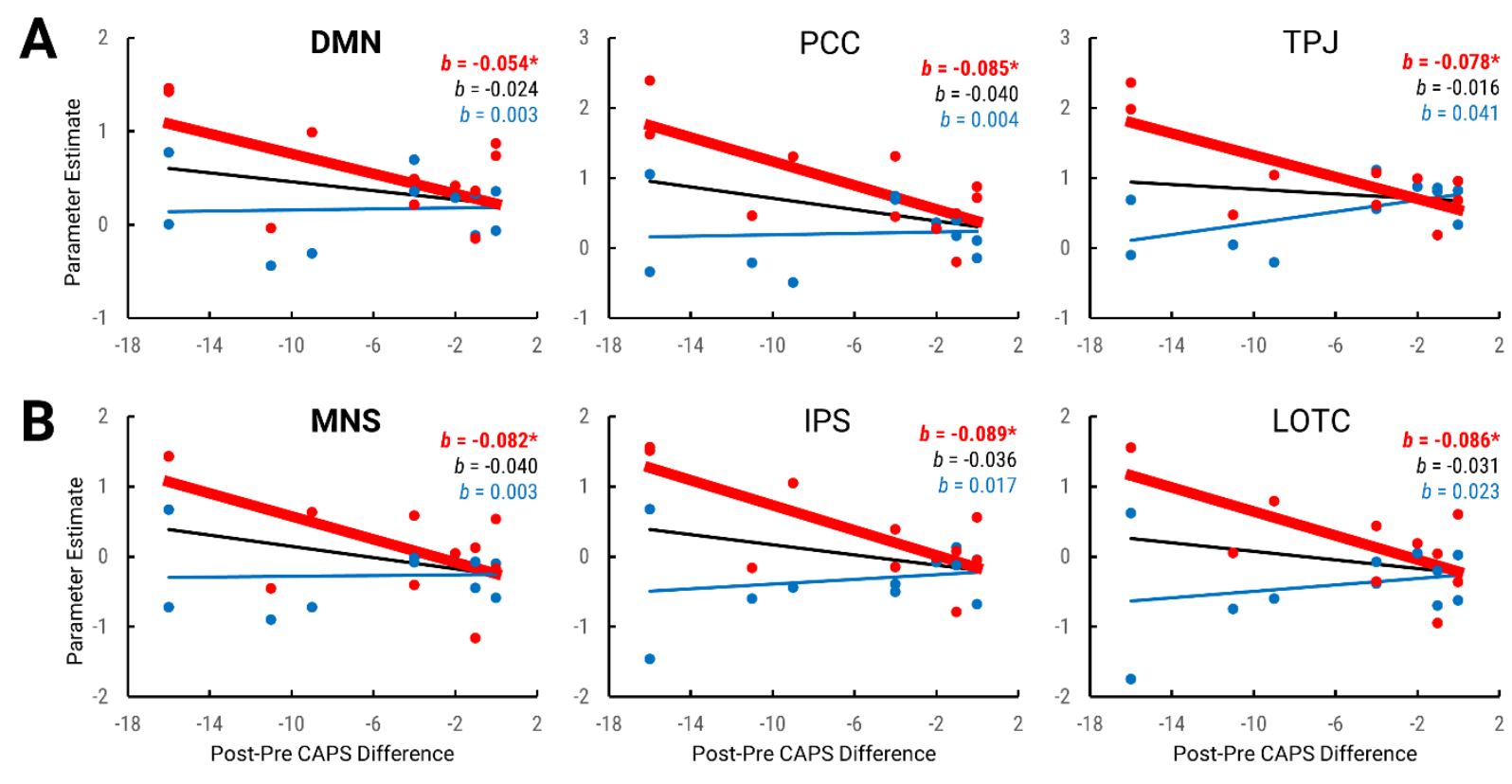

Emotions

Actions

Overall

Figure 5. Prediction of training outcomes from pre-training Why-How neural responses in PTSD patients who completed affect labeling training. (A) DMN and (B) MNS ROIs with significant predictive effects are shown. Scatterplots compare pre-training parameter estimates and Post-Pre CAPS differences, with regression lines plotted for main (overall) and simple (stimulus-specific) effects. Bolded lines represent significant regression coefficients, while thin lines represent non-significant regression coefficients. 
Table 3. Prediction of training outcomes (CAPSdiff) from pre-training Why-How neural responses in PTSD patients who completed affect labeling therapy.

\begin{tabular}{|c|c|c|c|c|c|c|c|c|c|c|}
\hline \multirow[b]{2}{*}{ Effect } & \multicolumn{5}{|c|}{ DMN ROIs } & \multicolumn{5}{|c|}{ MNS ROIs } \\
\hline & ROI & $\boldsymbol{b}$ & SE & $t$ & $p_{\mathrm{FDR}}$ & ROI & $\boldsymbol{b}$ & SE & $t$ & $p_{\mathrm{FDR}}$ \\
\hline CAPSdiff & DMN & -0.024 & 0.018 & -1.307 & 0.380 & MNS & -0.040 & 0.022 & -1.842 & 0.270 \\
\hline CAPSdiff $x$ Stimulus & & -0.030 & 0.009 & -3.309 & 0.005 & & -0.043 & 0.010 & -4.272 & 0.001 \\
\hline CAPSdiff (Emotions) $^{\dagger}$ & & -0.054 & 0.022 & -2.449 & 0.049 & & -0.082 & 0.030 & -2.753 & 0.036 \\
\hline CAPSdiff (Actions) ${ }^{\dagger}$ & & 0.003 & 0.019 & 0.153 & 0.987 & & 0.003 & 0.022 & 0.118 & 0.987 \\
\hline CAPSdiff & mPFC & -0.015 & 0.035 & -0.426 & 0.727 & plFG & -0.030 & 0.033 & -0.932 & 0.454 \\
\hline CAPSdiff $x$ Stimulus & & -0.023 & 0.014 & -1.654 & 0.127 & & -0.034 & 0.009 & -3.778 & 0.002 \\
\hline CAPSdiff (Emotions) $^{\dagger}$ & & & Intera & ction NS & & & -0.064 & 0.037 & -1.732 & 0.117 \\
\hline CAPSdiff (Actions) ${ }^{\dagger}$ & & & Intera & ction NS & & & 0.003 & 0.033 & 0.104 & 0.987 \\
\hline CAPSdiff & vmPFC & -0.005 & 0.015 & -0.354 & 0.727 & $\mathrm{dPMC}$ & -0.039 & 0.033 & -1.170 & 0.380 \\
\hline CAPSdiff $x$ Stimulus & & -0.010 & 0.011 & -0.942 & 0.358 & & -0.039 & 0.014 & -2.734 & 0.016 \\
\hline CAPSdiff (Emotions) ${ }^{\dagger}$ & & & Intera & ction NS & & & -0.077 & 0.039 & -1.971 & 0.092 \\
\hline CAPSdiff (Actions) ${ }^{\dagger}$ & & & Intera & ction NS & & & -0.001 & 0.038 & -0.017 & 0.987 \\
\hline CAPSdiff & PCC & -0.040 & 0.016 & -2.519 & 0.209 & IPS & -0.036 & 0.018 & -1.990 & 0.270 \\
\hline CAPSdiff $x$ Stimulus & & -0.051 & 0.013 & -3.954 & 0.002 & & -0.056 & 0.013 & -4.453 & 0.001 \\
\hline CAPSdiff (Emotions) ${ }^{\dagger}$ & & -0.085 & 0.024 & -3.503 & 0.013 & & -0.089 & 0.024 & -3.644 & 0.013 \\
\hline CAPSdiff (Actions) ${ }^{\dagger}$ & & 0.005 & 0.024 & 0.206 & 0.987 & & 0.017 & 0.026 & 0.643 & 0.987 \\
\hline CAPSdiff & TPJ & -0.017 & 0.015 & -1.146 & 0.380 & LOTC & -0.031 & 0.023 & -1.341 & 0.380 \\
\hline CAPSdiff $x$ Stimulus & & -0.050 & 0.010 & -5.273 & $<0.001$ & & -0.057 & 0.010 & -5.908 & $<0.001$ \\
\hline CAPSdiff (Emotions) ${ }^{\dagger}$ & & -0.078 & 0.021 & -3.704 & 0.013 & & -0.086 & 0.024 & -3.536 & 0.013 \\
\hline CAPSdiff (Actions) ${ }^{\dagger}$ & & 0.041 & 0.018 & 2.289 & 0.383 & & 0.023 & 0.029 & 0.798 & 0.987 \\
\hline
\end{tabular}

${ }^{\dagger}$ post-hoc simple effects test; CAPSdiff = Post-Pre CAPS score difference

\section{Discussion}

We conducted the first neuroimaging investigation of social inference in PTSD to help uncover the etiology of PTSD-related social cognitive impairments. To this end, we examined neural activation evoked by the Why/How social inference task (87), which dissociates the two primary networks of the "social brain": the default mode network (DMN) and mirror neuron system (MNS) $(25,51)$. We found that DMN and MNS responses differentiated PTSD patients from controls, correlated with symptom severity, and predicted training outcomes.

Unexpectedly, these effects were driven almost exclusively by hyperactivation to emotional stimuli. Our neuroimaging results were not corroborated by differences in Why/How task 


\section{PTSD AND THE SOCIAL BRAIN}

performance, despite numerous reports of impaired social inference performance in PTSD (18). This discrepancy may be attributable to the ease of the task. Taken together, these results suggest that the social brain may be inordinately selective for affective stimuli in PTSD, even in the absence of measurable behavioral impairments.

\section{Affect-Related Disruption of Social Inference Processing in PTSD}

To reveal PTSD-related disruptions in social inference processing, Why-How neural responses were analyzed for PTSD-control differences, symptom severity correlations, and prediction of training outcomes. In all three analyses, emotional facial expressions (Emotions) elicited much stronger PTSD-related effects than intentional hand actions (Actions). In the pretraining session, the PTSD group showed greater activation for Emotions, while controls showed greater activation for Actions. Critically, group differences were only significant for Emotions (Figure 3, Table 1). Similarly, pre-training symptom severity was positively correlated with Emotions-evoked activation and negatively correlated with Actions-evoked activation; these correlations were only significant for Emotions (Figure 4, Table 2). Lastly, better training outcomes were predicted by greater Emotions-evoked activation in the pre-training session (Figure 5, Table 3). These results were generally consistent throughout DMN and MNS, suggesting that both mentalizing and action identification processes are broadly selective for affective stimuli in PTSD. Taken together, affect-selective hyperactivation may be a defining characteristic of social inference processing in PTSD. This aligns with numerous reports of affect-selective hyperactivation in $\operatorname{PTSD}(68,81-83,86,110)$.

Given the overarching role of affect in our results, it would be reasonable to expect the strongest effects in ventromedial prefrontal cortex (vmPFC; Figure 2B), the hub of affective processing in the social brain $(21,111-113)$. Through a wide array of paradigms, vmPFC has been shown to compute the affective valence and value of social and non-social stimuli (114- 


\section{PTSD AND THE SOCIAL BRAIN}

119). During social inference, vmPFC can represent the emotions of others $(40,120-122)$. Unexpectedly, vmPFC did not show any significant PTSD-related effects in the current study, though the directionality of effects were consistent with other ROIs. Moreover, whole-brain analyses did not reveal significant PTSD-related effects in core affective regions such as amygdala (123), orbitofrontal cortex (124), and insula (125). Instead, all other ROIs featured significant PTSD-related effects, even though they are less implicated in affective processing (126). Thus, core affective processes may not play a key role in disrupting social inference processing in PTSD.

Alternatively, the affect-selective hyperactivation we observed may not reflect altered affect per se, but rather selective processing of affective information by the wide array of social cognitive processes subserved by DMN and MNS. Indeed, PTSD-related effects were stronger in the whole-network DMN and MNS masks, indicating that social inference processing was disrupted on a network-wide basis. Moreover, the within-network ROIs featured similar patterns of results as the whole-network masks, though effects were often weaker or not significant. Nevertheless, the consistency of these effects is remarkable given the functional heterogeneity between and within DMN and MNS $(122,127)$.

\section{An Attentional Account of Social Inference Dysfunction in PTSD}

Outside of core affective processes, what neurocognitive mechanisms could instigate such broad affect-selectivity throughout the social brain? Putatively, attention may be one such mechanism, as attentional processes are frequently reported to be inordinately biased towards emotional stimuli in PTSD $(82,77,128-136)$. Concordantly, PTSD-related attentional biases have been linked with affect-evoked hyperactivation throughout $\operatorname{DMN}(80,81)$. Moreover, DMN activation has been shown to correspond with attention level during social tasks $(48,88,137-$ 139). Similarly, MNS activation appears to be modulated by both top-down and bottom-up 


\section{PTSD AND THE SOCIAL BRAIN}

attention $(41,88,140-144)$.

An attentional account is further supported by the anatomical overlap between the attention networks and regions with significant PTSD-related effects in the present study. With the exception of medial prefrontal cortex, ROls with significant effects appear to overlap with either the dorsal attention (DAN), ventral attention (VAN), or frontoparietal control (FPCN) networks. DAN is involved in top-down attention, and includes intraparietal sulcus (IPS), dorsal premotor cortex (dPMC), and lateral occipitotemporal cortex $\operatorname{LOTC}(58,145,146)$. VAN, involved in bottom-up attention and attentional reorientation, includes temporoparietal junction (TPJ) and posterior inferior frontal gyrus $(\mathrm{pIFG)}(74,146)$. FPCN includes parts of posterior cingulate cortex (PCC), and is thought to facilitate attentional control by mediating activity between DMN, DAN, and other networks (147-150). Moreover, whole-brain analyses revealed PTSD-related effects in one region outside our a priori ROIs: the dorsolateral prefrontal cortex, a central node of DAN and FPCN $(145,151)$. Accordingly, other studies have observed affectevoked hyperactivation in DAN, VAN, and FPCN in PTSD $(76,80-83,85)$. Taken together, affective attentional biases in PTSD may drive widespread affect-selective hyperactivation throughout DMN and MNS during social inference.

\section{Affect Labeling Training}

The PTSD group underwent affect labeling training, which involves labeling the emotional content of stimuli (90). Affect labeling is an emotional inhibitory regulation strategy that has been found to downregulate amygdala responses via right ventrolateral prefrontal cortex (vIPFC) in healthy subjects $(90,91,152,153)$. Though we did not find PTSD-related effects in amygdala or vIPFC, affect labeling training was found to reduce symptom severity (94). Affect labeling may inhibit the affective components of social inference processing, as reactivity to emotional stimuli became negatively correlated with symptom severity after 


\section{PTSD AND THE SOCIAL BRAIN}

training-a reversal of the positive correlation found prior to training (Figure 4). This posttraining negative correlation reached significance only in pIFG, perhaps signifying the importance of mirroring and bottom-up attentional processes during socioaffective inhibitory regulation $(32,146)$. Moreover, better training outcomes were predicted by higher Emotionsevoked activation in the pre-training session (Figure 5), suggesting that engagement with emotional stimuli enhances the efficacy of affect labeling training-an interpretation consistent with other studies on exposure-based PTSD interventions (154-157). In sum, affect labeling training may be better suited for patients with greater affect-selective hyperactivation during social inference.

\section{Limitations and Future Directions}

The interpretation of these results should be tempered by the relatively small sample size of this study, especially in the post-training analyses. Additionally, generalizability may be limited by our selective recruitment of American veterans exposed to combat trauma. Future studies should use larger and more diverse samples. A potential confound in this study are the non-affective stimulus differences between Emotions (faces) and Actions (hands); our key finding of Emotions-selective hyperactivation may not be exclusively driven by affect. Future studies should better match emotional and non-emotional stimuli. Another caveat is the putative nature of the functional-anatomic overlap between our findings and the attention networks. This overlap was inferred using reverse inference from existing literature-a form of reasoning that can be tenuous $(158,159)$. This functional-anatomic overlap could be more definitively investigated by including functional localizers for DAN, VAN, and FPCN in addition to DMN and MNS. More broadly, techniques such as multivariate analyses, connectivity analyses, and other neuroimaging modalities would be useful in further characterizing PTSD-related neural dynamics during social inference. 


\section{PTSD AND THE SOCIAL BRAIN}

Conclusion

In the first neuroimaging investigation of social inference in PTSD, the social brain was found to be broadly selective towards emotional stimuli in PTSD. Affect-selective hyperactivation throughout DMN, MNS, and beyond differentiated PTSD patients from controls, correlated with symptom severity, and predicted training outcomes. Despite this, PTSD-related effects were not significant in core affective regions. Instead, our data putatively highlight the role of attentional processes in disrupting social inference processing in PTSD. These results indicate that further study of social inference processing in PTSD is strongly warranted, specifically in disentangling the roles of affect and attention.

\section{Acknowledgements}

This research was supported by the Defense Advanced Research Projects Agency

(DARPA), an agency of the U.S. Department of Defense, through the Army Research Laboratory (PIs: Burklund, Craske \& Lieberman; W911NF-14-C-0056). The author Kevin M. Tan was supported by the National Science Foundation Graduate Research Fellowship Program (DGE1650604).

\section{Disclosures}

The authors declare that the research was conducted in the absence of any commercial or financial relationships that could be construed as a potential conflict of interest. 


\section{PTSD AND THE SOCIAL BRAIN}

\section{References}

1. American Psychiatric Association (2013): Diagnostic and Statistical Manual of Mental Disorders, Fifth Edition. American Psychiatric Association. doi:

10.1176/appi.books.9780890425596.

2. Bagley C, MSW RR (1986): Sexual Abuse in Childhood: J Soc Work Hum Sex. 4: 33-47.

3. Alexander PC, Anderson CL, Brand B, Schaeffer CM, Grelling BZ, Kretz L (1998): Adult attachment and longterm effects in survivors of incest. Child Abuse Negl. 22: 45-61.

4. Stevens NR, Gerhart J, Goldsmith RE, Heath NM, Chesney SA, Hobfoll SE (2013): Emotion

Regulation Difficulties, Low Social Support, and Interpersonal Violence Mediate the Link Between Childhood Abuse and Posttraumatic Stress Symptoms. Behav Ther. 44: 152161.

5. Brewin CR, Andrews B, Valentine JD (2000): Meta-analysis of risk factors for posttraumatic stress disorder in trauma-exposed adults. J Consult Clin Psychol. 68: 748-766.

6. Nietlisbach G, Maercker A (2009): Social Cognition and Interpersonal Impairments in Trauma Survivors with PTSD. J Aggress Maltreatment Trauma. 18: 382-402.

7. Sharp C, Fonagy P, Allen JG (2012): Posttraumatic Stress Disorder: A Social-Cognitive Perspective. Clin Psychol Sci Pract. 19: 229-240.

8. Knežević M, Jovančević M (2004): The IFEEL pictures: Psychological trauma and perception, and interpretation of child's emotions. Nord J Psychiatry. 58: 139-145.

9. Shin LM, Wright Cl, Cannistraro PA, Wedig MM, McMullin K, Martis B, et al. (2005): A Functional Magnetic Resonance Imaging Study of Amygdala and Medial Prefrontal Cortex Responses to Overtly Presented Fearful Faces in Posttraumatic Stress Disorder. Arch Gen Psychiatry. 62: 273-281.

10. Schmidt JZ, Zachariae R (2009): PTSD and Impaired Eye Expression Recognition: A Preliminary Study. J Loss Trauma. 14: 46-56. 


\section{PTSD AND THE SOCIAL BRAIN}

11. Fonzo GA, Simmons AN, Thorp SR, Norman SB, Paulus MP, Stein MB (2010): Exaggerated and Disconnected Insular-Amygdalar Blood Oxygenation Level-Dependent Response to Threat-Related Emotional Faces in Women with Intimate-Partner Violence Posttraumatic Stress Disorder. Biol Psychiatry, Stress, Neuroplasticity, and Posttraumatic Stress Disorder. 68: 433-441.

12. Poljac E, Montagne B, de Haan EHF (2011): Reduced recognition of fear and sadness in post-traumatic stress disorder. Cortex. 47: 974-980.

13. Mazza M, Giusti L, Albanese A, Mariano M, Pino MC, Roncone R (2012): Social cognition disorders in military police officers affected by posttraumatic stress disorder after the attack of An-Nasiriyah in Iraq 2006. Psychiatry Res. 198: 248-252.

14. Allen JG, Fonagy P (2006): The Handbook of Mentalization-Based Treatment. John Wiley \& Sons.

15. Nietlisbach G, Maercker A, Rösler W, Haker H (2010): Are Empathic Abilities Impaired in Posttraumatic Stress Disorder? Psychol Rep. 106: 832-844.

16. Nazarov A, Frewen P, Parlar M, Oremus C, MacQueen G, McKinnon M, Lanius R (2014): Theory of mind performance in women with posttraumatic stress disorder related to childhood abuse. Acta Psychiatr Scand. 129: 193-201.

17. Parlar M, Frewen P, Nazarov A, Oremus C, MacQueen G, Lanius R, McKinnon MC (2014): Alterations in empathic responding among women with posttraumatic stress disorder associated with childhood trauma. Brain Behav. 4: 381-389.

18. Plana I, Lavoie M-A, Battaglia M, Achim AM (2014): A meta-analysis and scoping review of social cognition performance in social phobia, posttraumatic stress disorder and other anxiety disorders. J Anxiety Disord. 28: 169-177.

19. Premack D, Woodruff G (1978): Does the chimpanzee have a theory of mind? Behav Brain Sci. 1: 515. 


\section{PTSD AND THE SOCIAL BRAIN}

20. Schaafsma SM, Pfaff DW, Spunt RP, Adolphs R (2015): Deconstructing and reconstructing theory of mind. Trends Cogn Sci. 19: 65-72.

21. Mitchell RLC, Phillips LH (2015): The overlapping relationship between emotion perception and theory of mind. Neuropsychologia. 70: 1-10.

22. Rizzolatti G, Craighero L (2004): The Mirror-Neuron System. Annu Rev Neurosci. 27: 169192.

23. Buckner RL, Andrews-Hanna JR, Schacter DL (2008): The Brain's Default Network. Ann N Y Acad Sci. 1124: 1-38.

24. Mars RB, Neubert F-X, Noonan MP, Sallet J, Toni I, Rushworth MFS (2012): On the relationship between the "default mode network" and the "social brain." Front Hum Neurosci. 6: 189.

25. Spunt RP, Falk EB, Lieberman MD (2010): Dissociable Neural Systems Support Retrieval of How and Why Action Knowledge. Psychol Sci. 21: 1593-1598.

26. Waytz A, Mitchell JP (2011): Two Mechanisms for Simulating Other Minds Dissociations Between Mirroring and Self-Projection. Curr Dir Psychol Sci. 20: 197-200.

27. Spunt RP, Satpute AB, Lieberman MD (2011): Identifying the What, Why, and How of an Observed Action: An fMRI Study of Mentalizing and Mechanizing during Action Observation. J Cogn Neurosci. 23: 63-74.

28. Spunt RP, Kemmerer D, Adolphs R (2016): The neural basis of conceptualizing the same action at different levels of abstraction. Soc Cogn Affect Neurosci. 11: 1141-1151.

29. di Pellegrino G, Fadiga L, Fogassi L, Gallese V, Rizzolatti G (1992): Understanding motor events: a neurophysiological study. Exp Brain Res. 91: 176-180.

30. Rizzolatti G, Fadiga L, Gallese V, Fogassi L (1996): Premotor cortex and the recognition of motor actions. Cogn Brain Res, Mental representations of motor acts. 3: 131-141. 


\section{PTSD AND THE SOCIAL BRAIN}

31. Gallese V, Fadiga L, Fogassi L, Rizzolatti G (1996): Action recognition in the premotor cortex. Brain. 119: 593-609.

32. Oosterhof NN, Tipper SP, Downing PE (2013): Crossmodal and action-specific: neuroimaging the human mirror neuron system. Trends Cogn Sci. 17: 311-318.

33. Buccino G, Binkofski F, Fink GR, Fadiga L, Fogassi L, Gallese V, et al. (2001): Action observation activates premotor and parietal areas in a somatotopic manner: an fMRI study. Eur J Neurosci. 13: 400-404.

34. de Gelder B, Van den Stock J, Meeren HKM, Sinke CBA, Kret ME, Tamietto M (2010): Standing up for the body. Recent progress in uncovering the networks involved in the perception of bodies and bodily expressions. Neurosci Biobehav Rev. 34: 513-527.

35. Grosbras M-H, Paus T (2006): Brain Networks Involved in Viewing Angry Hands or Faces. Cereb Cortex. 16: 1087-1096.

36. Urgesi C, Candidi M, Avenanti A (2014): Neuroanatomical substrates of action perception and understanding: an anatomic likelihood estimation meta-analysis of lesion-symptom mapping studies in brain injured patients. Front Hum Neurosci. 8. doi: 10.3389/fnhum.2014.00344.

37. Kemmerer D (2015): Are the motor features of verb meanings represented in the precentral motor cortices? Yes, but within the context of a flexible, multilevel architecture for conceptual knowledge. Psychon Bull Rev. 22: 1068-1075.

38. Keysers C, Gazzola V (2007): Integrating simulation and theory of mind: from self to social cognition. Trends Cogn Sci. 11: 194-196.

39. Zaki J, Weber J, Bolger N, Ochsner K (2009): The neural bases of empathic accuracy. Proc Natl Acad Sci. 106: 11382-11387.

40. Spunt RP, Lieberman MD (2012): An integrative model of the neural systems supporting the comprehension of observed emotional behavior. Neurolmage. 59: 3050-3059. 


\section{PTSD AND THE SOCIAL BRAIN}

41. Spunt RP, Lieberman MD (2012): Dissociating Modality-Specific and Supramodal Neural Systems for Action Understanding. J Neurosci. 32: 3575-3583.

42. Mossad SI, AuCoin-Power M, Urbain C, Smith ML, Pang EW, Taylor MJ (2016): Thinking about the thoughts of others; temporal and spatial neural activation during false belief reasoning. Neurolmage. 134: 320-327.

43. Zhang L, Gan JQ, Zheng W, Wang H (2018): Spatiotemporal Phase Synchronization in Adaptive Reconfiguration from Action Observation Network to Mentalizing Network for Understanding Other's Action Intention. Brain Topogr. 31: 447-467.

44. Frith CD, Frith U (2006): The Neural Basis of Mentalizing. Neuron. 50: 531-534.

45. Saxe R, Powell LJ (2006): It's the Thought That Counts: Specific Brain Regions for One Component of Theory of Mind. Psychol Sci. 17: 692-699.

46. Zaki J, Ochsner KN (2012): The neuroscience of empathy: progress, pitfalls and promise. Nat Neurosci. 15: 675-680.

47. Reniers RLEP, Corcoran R, Völlm BA, Mashru A, Howard R, Liddle PF (2012): Moral decisionmaking, ToM, empathy and the default mode network. Biol Psychol. 90: 202-210.

48. Meyer ML, Spunt RP, Berkman ET, Taylor SE, Lieberman MD (2012): Evidence for social working memory from a parametric functional MRI study. Proc Natl Acad Sci. 109: $1883-1888$.

49. Davey CG, Pujol J, Harrison BJ (2016): Mapping the self in the brain's default mode network. Neurolmage. 132: 390-397.

50. Schilbach L, Eickhoff SB, Rotarska-Jagiela A, Fink GR, Vogeley K (2008): Minds at rest? Social cognition as the default mode of cognizing and its putative relationship to the "default system" of the brain. Conscious Cogn, Social Cognition, Emotion, and SelfConsciousness. 17: 457-467. 


\section{PTSD AND THE SOCIAL BRAIN}

51. Lieberman MD (2010): Social Cognitive Neuroscience. Handb Soc Psychol. American Cancer Society. doi: 10.1002/9780470561119.socpsy001005.

52. Amft M, Bzdok D, Laird AR, Fox PT, Schilbach L, Eickhoff SB (2015): Definition and characterization of an extended social-affective default network. Brain Struct Funct. 220: $1031-1049$.

53. Li W, Mai X, Liu C (2014): The default mode network and social understanding of others: what do brain connectivity studies tell us. Front Hum Neurosci. 8. doi: 10.3389/fnhum.2014.00074.

54. Andrews-Hanna JR (2012): The Brain's Default Network and Its Adaptive Role in Internal Mentation. The Neuroscientist. 18: 251-270.

55. Ingvar DH, Schwartz MS (n.d.): BLOOD FLOW PATTERNS INDUCED IN THE DOMINANT HEMISPHERE BY SPEECH AND READING. 16.

56. Shulman GL, Fiez JA, Corbetta M, Buckner RL, Miezin FM, Raichle ME, Petersen SE (1997): Common Blood Flow Changes across Visual Tasks: II. Decreases in Cerebral Cortex. J Cogn Neurosci. 9: 648-663.

57. Raichle ME, MacLeod AM, Snyder AZ, Powers WJ, Gusnard DA, Shulman GL (2001): A default mode of brain function. Proc Natl Acad Sci. 98: 676-682.

58. Fox MD, Corbetta M, Snyder AZ, Vincent JL, Raichle ME (2006): Spontaneous neuronal activity distinguishes human dorsal and ventral attention systems. Proc Natl Acad Sci. 103: $10046-10051$.

59. Raichle ME, Snyder AZ (2007): A default mode of brain function: a brief history of an evolving idea. Neurolmage. 37: 1083-1090; discussion 1097-1099.

60. Lanius R, Frewen PA, Nazarov A, McKinnon MC (2014): A Social-Cognitive-Neuroscience Approach to PTSD: Clinical and Research Perspectives. In: Lanius UF, Paulsen SL, 


\section{PTSD AND THE SOCIAL BRAIN}

Corrigan FM, editors. Neurobiol Treat Trauma Dissociation. New York, NY: Springer Publishing Company. doi: 10.1891/9780826106322.0004.

61. Frewen P, Lane RD, Neufeld RWJ, Densmore M, Stevens T, Lanius R (2008): Neural Correlates of Levels of Emotional Awareness During Trauma Script-Imagery in Posttraumatic Stress Disorder. Psychosom Med. 70: 27.

62. Frewen PA, Dozois DJA, Neufeld RWJ, Densmore M, Stevens TK, Lanius RA (2010): Social emotions and emotional valence during imagery in women with PTSD: Affective and neural correlates. Psychol Trauma Theory Res Pract Policy. 2: 145-157.

63. Frewen PA, Dozois DJA, Neufeld RWJ, Lane RD, Densmore M, Stevens TK, Lanius RA (2012): Emotional Numbing in Posttraumatic Stress Disorder: A Functional Magnetic Resonance Imaging Study. J Clin Psychiatry. 73: 431-436.

64. Frewen PA, Dozois DJA, Neufeld RWJ, Densmore M, Stevens TK, Lanius RA (2011): Selfreferential processing in women with PTSD: Affective and neural response. Psychol Trauma Theory Res Pract Policy. 3: 318-328.

65. Bluhm RL, Frewen PA, Coupland NC, Densmore M, Schore AN, Lanius RA (2012): Neural correlates of self-reflection in post-traumatic stress disorder: Neurobiology of selfreflection in PTSD. Acta Psychiatr Scand. 125: 238-246.

66. Frewen P, Thornley E, Rabellino D, Lanius R (2017): Neuroimaging the traumatized self: fMRI reveals altered response in cortical midline structures and occipital cortex during visual and verbal self- and other-referential processing in women with PTSD. Eur $J$ Psychotraumatology. 8: 1314164.

67. Cisler JM, Scott Steele J, Smitherman S, Lenow JK, Kilts CD (2013): Neural processing correlates of assaultive violence exposure and PTSD symptoms during implicit threat processing: A network-level analysis among adolescent girls. Psychiatry Res Neuroimaging. 214: 238-246. 


\section{PTSD AND THE SOCIAL BRAIN}

68. Rabellino D, Tursich M, Frewen PA, Daniels JK, Densmore M, Théberge J, Lanius RA (2015): Intrinsic Connectivity Networks in post-traumatic stress disorder during sub- and supraliminal processing of threat-related stimuli. Acta Psychiatr Scand. 132: 365-378.

69. Bluhm RL, Williamson PC, Osuch EA, Frewen PA, Stevens TK, Boksman K, et al. (2009): Alterations in default network connectivity in posttraumatic stress disorder related to early-life trauma. J Psychiatry Neurosci. 8.

70. Sripada RK, King AP, Welsh RC, Garfinkel SN, Wang X, Sripada CS, Liberzon I (2012): Neural Dysregulation in Posttraumatic Stress Disorder: Evidence for Disrupted Equilibrium Between Salience and Default Mode Brain Networks. Psychosom Med. 74: 904-911.

71. Tursich M, Ros T, Frewen PA, Kluetsch RC, Calhoun VD, Lanius RA (2015): Distinct intrinsic network connectivity patterns of post-traumatic stress disorder symptom clusters. Acta Psychiatr Scand. 132: 29-38.

72. Zhang Y, Liu F, Chen H, Li M, Duan X, Xie B, Chen H (2015): Intranetwork and internetwork functional connectivity alterations in post-traumatic stress disorder. J Affect Disord. 187: $114-121$.

73. DiGangi JA, Tadayyon A, Fitzgerald DA, Rabinak CA, Kennedy A, Klumpp H, et al. (2016): Reduced default mode network connectivity following combat trauma. Neurosci Lett. 615: $37-43$.

74. Corbetta M, Patel G, Shulman GL (2008): The Reorienting System of the Human Brain: From Environment to Theory of Mind. Neuron. 58: 306-324.

75. Molenberghs $P$, Cunnington R, Mattingley JB (2012): Brain regions with mirror properties: $A$ meta-analysis of 125 human fMRI studies. Neurosci Biobehav Rev. 36: 341-349.

76. Block SR, Liberzon I (2016): Attentional processes in posttraumatic stress disorder and the associated changes in neural functioning. Exp Neurol. 284: 153-167. 


\section{PTSD AND THE SOCIAL BRAIN}

77. Vasterling JJ, Brailey K, Constans JI, Sutker PB (1998): Attention and memory dysfunction in posttraumatic stress disorder. Neuropsychology. 12: 125-133.

78. Shin LM, Whalen PJ, Pitman RK, Bush G, Macklin ML, Lasko NB, et al. (2001): An fMRI study of anterior cingulate function in posttraumatic stress disorder. Biol Psychiatry. 50: 932942.

79. Bremner JD, Vermetten E, Vythilingam M, Afzal N, Schmahl C, Elzinga B, Charney DS (2004): Neural correlates of the classic color and emotional stroop in women with abuse-related posttraumatic stress disorder. Biol Psychiatry. 55: 612-620.

80. Morey RA, Petty CM, Cooper DA, LaBar KS, McCarthy G (2008): Neural systems for executive and emotional processing are modulated by symptoms of posttraumatic stress disorder in Iraq War veterans. Psychiatry Res Neuroimaging. 162: 59-72.

81. Hayes JP, LaBar KS, Petty CM, McCarthy G, Morey RA (2009): Alterations in the neural circuitry for emotion and attention associated with posttraumatic stress symptomatology. Psychiatry Res Neuroimaging. 172: 7-15.

82. Fani N, Jovanovic T, Ely TD, Bradley B, Gutman D, Tone EB, Ressler KJ (2012): Neural correlates of attention bias to threat in post-traumatic stress disorder. Biol Psychol. 90: $134-142$.

83. Thomaes K, Dorrepaal E, Draijer N, Ruiter D, B M, Elzinga BM, et al. (2013): Increased anterior cingulate cortex and hippocampus activation in Complex PTSD during encoding of negative words. Soc Cogn Affect Neurosci. 8: 190-200.

84. Blair KS, Vythilingam M, Crowe SL, McCaffrey DE, Ng P, Wu CC, et al. (2013): Cognitive control of attention is differentially affected in trauma-exposed individuals with and without post-traumatic stress disorder. Psychol Med. 43: 85-95. 


\section{PTSD AND THE SOCIAL BRAIN}

85. White SF, Costanzo ME, Blair JR, Roy MJ (2015): PTSD symptom severity is associated with increased recruitment of top-down attentional control in a trauma-exposed sample. Neurolmage Clin. 7: 19-27.

86. Khanna MM, Badura-Brack AS, McDermott TJ, Embury CM, Wiesman Al, Shepherd A, et al. (2017): Veterans with post-traumatic stress disorder exhibit altered emotional processing and attentional control during an emotional Stroop task. Psychol Med. 47: 2017-2027.

87. Spunt RP, Adolphs R (2014): Validating the Why/How contrast for functional MRI studies of Theory of Mind. Neurolmage. 99: 301-311.

88. Spunt RP, Lieberman MD (2013): The Busy Social Brain: Evidence for Automaticity and Control in the Neural Systems Supporting Social Cognition and Action Understanding. Psychol Sci. 24: 80-86.

89. Blake DD, Weathers FW, Nagy LM, Kaloupek DG, Gusman FD, Charney DS, Keane TM (1995): The development of a Clinician-Administered PTSD Scale. J Trauma Stress. 8: 75-90. 90. Lieberman MD, Eisenberger NI, Crockett MJ, Tom SM, Pfeifer JH, Way BM (2007): Putting Feelings Into Words. Psychol Sci. 18: 421-428.

91. Burklund LJ, Creswell JD, Irwin MR, Lieberman MD (2014): The common and distinct neural bases of affect labeling and reappraisal in healthy adults. Front Psychol. 5. doi: 10.3389/fpsyg.2014.00221.

92. Constantinou E, Van Den Houte M, Bogaerts K, Van Diest I, Van den Bergh O (2014): Can words heal? Using affect labeling to reduce the effects of unpleasant cues on symptom reporting. Front Psychol. 5. doi: 10.3389/fpsyg.2014.00807.

93. Torre JB, Lieberman MD (2018): Putting Feelings Into Words: Affect Labeling as Implicit Emotion Regulation. Emot Rev. 10: 116-124. 


\section{PTSD AND THE SOCIAL BRAIN}

94. Burklund LJ, Carolyn DD, Niles AN, Torre JB, Brown L, Vinograd M, et al. (Under review):

Affect Labeling: A Promising New Approach to Treating Combat-Related PTSD in Veterans. .

95. Spunt R (n.d.): Why/How Localizer. . Retrieved September 26, 2018, from http://www.bobspunt.com/whyhowlocalizer/.

96. Kleiner M, Brainard D, Pelli D, Ingling A, Murray R, Broussard C, others (2007): What's new in Psychtoolbox-3. Perception. 36: 1.

97. Mathworks (2007): Matlab R2007. . Retrieved September 25, 2018, from https://www.mathworks.com/help/matlab/release-notes-R2007b.html.

98. Friston K, John Ashburner, Stefan Kiebel, Thomas Nichols, William Penny (2007): Statistical Parametric Mapping. Elsevier. doi: 10.1016/B978-0-12-372560-8.X5000-1.

99. Ashburner J (2007): A fast diffeomorphic image registration algorithm. Neurolmage. 38: 95113.

100. Collins DL, Zijdenbos AP, Kollokian V, Sled JG, Kabani NJ, Holmes CJ, Evans AC (1998): Design and construction of a realistic digital brain phantom. IEEE Trans Med Imaging. 17: $463-468$.

101. Mathworks (2016): R2016b - Updates to the MATLAB and Simulink product families. . Retrieved September 25, 2018, from https://www.mathworks.com/products/new_products/release2016b.html.

102. Barr DJ, Levy R, Scheepers C, Tily HJ (2013): Random effects structure for confirmatory hypothesis testing: Keep it maximal. J Mem Lang. 68: 255-278.

103. Barr DJ (2013): Random effects structure for testing interactions in linear mixed-effects models. Front Psychol. 4. doi: 10.3389/fpsyg.2013.00328.

104. Hardy MA (1993): Regression with dummy variables. Regression with dummy variables. Thousand Oaks, CA, US: Sage Publications, Inc. 


\section{PTSD AND THE SOCIAL BRAIN}

105. Andrews-Hanna JR, Reidler JS, Sepulcre J, Poulin R, Buckner RL (2010): FunctionalAnatomic Fractionation of the Brain's Default Network. Neuron. 65: 550-562.

106. Benjamini $Y$, Hochberg $Y$ (1995): Controlling the false discovery rate: a practical and powerful approach to multiple testing. J R Stat Soc Ser B Methodol. 289-300.

107. Yekutieli D, Benjamini Y (1999): Resampling-based false discovery rate controlling multiple test procedures for correlated test statistics. J Stat Plan Inference. 82: 171-196.

108. Shultz AP (2018, March 17): MR Tools. . Retrieved September 25, 2018, from http://mrtools.mgh.harvard.edu/index.php?title=Main_Page.

109. Cox RW, Chen G, Glen DR, Reynolds RC, Taylor PA (2017): FMRI Clustering in AFNI: FalsePositive Rates Redux. Brain Connect. 7: 152-171.

110. MacNamara A, Post D, Kennedy AE, Rabinak CA, Phan KL (2013): Electrocortical processing of social signals of threat in combat-related post-traumatic stress disorder. Biol Psychol. 94: 441-449.

111. Shamay-Tsoory SG, Tibi-Elhanany Y, Aharon-Peretz J (2006): The ventromedial prefrontal cortex is involved in understanding affective but not cognitive theory of mind stories. Soc Neurosci. 1: 149-166.

112. Shamay-Tsoory SG, Aharon-Peretz J (2007): Dissociable prefrontal networks for cognitive and affective theory of mind: A lesion study. Neuropsychologia. 45: 3054-3067.

113. Schlaffke L, Lissek S, Lenz M, Juckel G, Schultz T, Tegenthoff M, et al. (2015): Shared and nonshared neural networks of cognitive and affective theory-of-mind: A neuroimaging study using cartoon picture stories. Hum Brain Mapp. 36: 29-39.

114. Winecoff A, Clithero JA, Carter RM, Bergman SR, Wang L, Huettel SA (2013): Ventromedial Prefrontal Cortex Encodes Emotional Value. J Neurosci. 33: 11032-11039.

115. Chikazoe J, Lee DH, Kriegeskorte N, Anderson AK (2014): Population coding of affect across stimuli, modalities and individuals. Nat Neurosci. 17: 1114-1122. 


\section{PTSD AND THE SOCIAL BRAIN}

116. Skerry AE, Saxe R (2015): Neural Representations of Emotion Are Organized around Abstract Event Features. Curr Biol. 25: 1945-1954.

117. Hare TA, Camerer CF, Rangel A (2009): Self-Control in Decision-Making Involves Modulation of the vmPFC Valuation System. Science. 324: 646-648.

118. Hare TA, Camerer CF, Knoepfle DT, O’Doherty JP, Rangel A (2010): Value Computations in Ventral Medial Prefrontal Cortex during Charitable Decision Making Incorporate Input from Regions Involved in Social Cognition. J Neurosci. 30: 583-590.

119. Chib VS, Rangel A, Shimojo S, O'Doherty JP (2009): Evidence for a Common Representation of Decision Values for Dissimilar Goods in Human Ventromedial Prefrontal Cortex. $J$ Neurosci. 29: 12315-12320.

120. Corradi-Dell'Acqua C, Hofstetter C, Vuilleumier P (2014): Cognitive and affective theory of mind share the same local patterns of activity in posterior temporal but not medial prefrontal cortex. Soc Cogn Affect Neurosci. 9: 1175-1184.

121. Tamir DI, Thornton MA, Contreras JM, Mitchell JP (2016): Neural evidence that three dimensions organize mental state representation: Rationality, social impact, and valence. Proc Natl Acad Sci. 113: 194-199.

122. Koster-Hale J, Richardson H, Velez N, Asaba M, Young L, Saxe R (2017): Mentalizing regions represent distributed, continuous, and abstract dimensions of others' beliefs. Neurolmage. 161: 9-18.

123. Phelps EA, LeDoux JE (2005): Contributions of the Amygdala to Emotion Processing: From Animal Models to Human Behavior. Neuron. 48: 175-187.

124. Rolls ET, Grabenhorst F (2008): The orbitofrontal cortex and beyond: From affect to decision-making. Prog Neurobiol. 86: 216-244.

125. Singer T, Critchley HD, Preuschoff K (2009): A common role of insula in feelings, empathy and uncertainty. Trends Cogn Sci. 13: 334-340. 


\section{PTSD AND THE SOCIAL BRAIN}

126. Lindquist KA, Wager TD, Kober H, Bliss-Moreau E, Barrett LF (2012): The brain basis of emotion: A meta-analytic review. Behav Brain Sci. 35: 121-143.

127. Handjaras G, Bernardi G, Benuzzi F, Nichelli PF, Pietrini P, Ricciardi E (2015): A topographical organization for action representation in the human brain. Hum Brain Mapp. 36: 3832-3844.

128. MacLeod C, Mathews A, Tata P (1986): Attentional bias in emotional disorders. J Abnorm Psychol. 95: 15-20.

129. Foa EB, Feske U, Murdock TB, Kozak MJ, McCarthy PR (1991): Processing of threat-related information in rape victims. J Abnorm Psychol. 100: 156-162.

130. Litz BT, Weathers FW, Monaco V, Herman DS, Wulfsohn M, Marx B, Keane TM (1996): Attention, arousal, and memory in posttraumatic stress disorder. J Trauma Stress. 9: 497-519.

131. Bryant RA, Harvey AG (1995): Processing threatening information in posttraumatic stress disorder. J Abnorm Psychol. 104: 537-541.

132. Dalgleish T, Taghavi R, Neshat-Doost H, Moradi A, Canterbury R, Yule W (2003): Patterns of Processing Bias for Emotional Information Across Clinical Disorders: A Comparison of Attention, Memory, and Prospective Cognition in Children and Adolescents With Depression, Generalized Anxiety, and Posttraumatic Stress Disorder. J Clin Child Adolesc Psychol. 32: 10-21.

133. Elsesser K, Sartory G, Tackenberg A (2004): Attention, Heart Rate, and Startle Response During Exposure to Trauma-Relevant Pictures: A Comparison of Recent Trauma Victims and Patients With Posttraumatic Stress Disorder. J Abnorm Psychol. 113: 289-301.

134. Vythilingam M, Blair KS, McCAFFREY D, Scaramozza M, Jones M, Nakic M, et al. (2007):

Biased emotional attention in post-traumatic stress disorder: a help as well as a hindrance? Psychol Med. 37: 1445-1455. 


\section{PTSD AND THE SOCIAL BRAIN}

135. Bardeen JR, Orcutt HK (2011): Attentional control as a moderator of the relationship between posttraumatic stress symptoms and attentional threat bias. J Anxiety Disord. 25: 1008-1018.

136. Fani N, Tone EB, Phifer J, Norrholm SD, Bradley B, Ressler KJ, et al. (2012): Attention bias toward threat is associated with exaggerated fear expression and impaired extinction in PTSD. Psychol Med. 42: 533-543.

137. den Ouden HEM, Frith U, Frith C, Blakemore S-J (2005): Thinking about intentions. Neurolmage, Special Section: Social Cognitive Neuroscience. 28: 787-796.

138. Rameson LT, Morelli SA, Lieberman MD (2011): The Neural Correlates of Empathy: Experience, Automaticity, and Prosocial Behavior. J Cogn Neurosci. 24: 235-245.

139. Wagner DD, Kelley WM, Heatherton TF (2011): Individual Differences in the Spontaneous Recruitment of Brain Regions Supporting Mental State Understanding When Viewing Natural Social Scenes. Cereb Cortex. 21: 2788-2796.

140. Engel A, Burke M, Fiehler K, Bien S, Rösler F (2008): What activates the human mirror neuron system during observation of artificial movements: Bottom-up visual features or top-down intentions? Neuropsychologia, Part Special Issue: What is the Parietal Lobe Contribution to Human Memory? 46: 2033-2042.

141. Hesse MD, Sparing R, Fink GR (2008): End or Means-The "What" and "How" of Observed Intentional Actions. J Cogn Neurosci. 21: 776-790.

142. Wang H, Callaghan E, Gooding-Williams G, McAllister C, Kessler K (2016): Rhythm makes the world go round: An MEG-TMS study on the role of right TPJ theta oscillations in embodied perspective taking. Cortex. 75: 68-81.

143. Kilner JM, Marchant JL, Frith CD (2006): Modulation of the mirror system by social relevance. Soc Cogn Affect Neurosci. 1: 143-148. 


\section{PTSD AND THE SOCIAL BRAIN}

144. Buccino G, Baumgaertner A, Colle L, Buechel C, Rizzolatti G, Binkofski F (2007): The neural basis for understanding non-intended actions. Neurolmage, Cortical Control of Higher Motor Cognition. 36: T119-T127.

145. Corbetta M, Shulman GL (2002): Control of goal-directed and stimulus-driven attention in the brain. Nat Rev Neurosci. 3: 201-215.

146. Vossel S, Geng JJ, Fink GR (2014): Dorsal and Ventral Attention Systems: Distinct Neural Circuits but Collaborative Roles. The Neuroscientist. 20: 150-159.

147. Spreng RN, Stevens WD, Chamberlain JP, Gilmore AW, Schacter DL (2010): Default network activity, coupled with the frontoparietal control network, supports goal-directed cognition. Neurolmage. 53: 303-317.

148. Leech R, Braga R, Sharp DJ (2012): Echoes of the Brain within the Posterior Cingulate Cortex. J Neurosci. 32: 215-222.

149. Leech R, Sharp DJ (2014): The role of the posterior cingulate cortex in cognition and disease. Brain. 137: 12-32.

150. Dixon ML, Vega ADL, Mills C, Andrews-Hanna J, Spreng RN, Cole MW, Christoff K (2018): Heterogeneity within the frontoparietal control network and its relationship to the default and dorsal attention networks. Proc Natl Acad Sci. 201715766.

151. Harding IH, Yücel M, Harrison BJ, Pantelis C, Breakspear M (2015): Effective connectivity within the frontoparietal control network differentiates cognitive control and working memory. Neurolmage. 106: 144-153.

152. Torrisi SJ, Lieberman MD, Bookheimer SY, Altshuler LL (2013): Advancing understanding of affect labeling with dynamic causal modeling. Neurolmage. 82: 481-488.

153. Hariri AR, Bookheimer SY, Mazziotta JC (2000): Modulating emotional responses: effects of a neocortical network on the limbic system. NeuroReport. 11: 43. 


\section{PTSD AND THE SOCIAL BRAIN}

154. Jaycox LH, Foa EB, Morral AR (1998): Influence of emotional engagement and habituation on exposure therapy for PTSD. J Consult Clin Psychol. 66: 185-192.

155. Craske MG, Kircanski K, Zelikowsky M, Mystkowski J, Chowdhury N, Baker A (2008):

Optimizing inhibitory learning during exposure therapy. Behav Res Ther. 46: 5-27.

156. Niles AN, Mesri B, Burklund LJ, Lieberman MD, Craske MG (2013): Attentional bias and emotional reactivity as predictors and moderators of behavioral treatment for social phobia. Behav Res Ther. 51: 669-679.

157. Barry TJ, Sewart AR, Arch JJ, Craske MG (2015): Deficits in Disengaging Attention from Threat Predict Improved Response to Cognitive Behavioral Therapy for Anxiety. Depress Anxiety. 32: 892-899.

158. Poldrack RA (2006): Can cognitive processes be inferred from neuroimaging data? Trends Cogn Sci. 10: 59-63.

159. Aguirre GK, Feinberg FTE, Farah (eds MJ, Neurology B (2003): Functional imaging in behavioral neurology and cognitive neuropsychology. Behav Neurol Cogn Neuropsychol 2nd Ed. McGraw Hill. 